\title{
Logic and Lattices for Distributed Programming
}

\author{
Neil Conway \\ UC Berkeley \\ nrc@cs.berkeley.edu \\ Joseph M. Hellerstein \\ UC Berkeley \\ hellerstein@cs.berkeley.edu
}

\author{
William R. Marczak \\ UC Berkeley \\ wrm@cs.berkeley.edu
}

\author{
Peter Alvaro \\ UC Berkeley \\ palvaro@cs.berkeley.edu
}

\author{
David Maier \\ Portland State University \\ maier@cs.pdx.edu
}

\begin{abstract}
In recent years there has been interest in achieving application-level consistency criteria without the latency and availability costs of strongly consistent storage infrastructure. A standard technique is to adopt a vocabulary of commutative operations; this avoids the risk of inconsistency due to message reordering. Another approach was recently captured by the CALM theorem, which proves that logically monotonic programs are guaranteed to be eventually consistent. In logic languages such as Bloom, CALM analysis can automatically verify that programs achieve consistency without coordination.

In this paper we present $\mathrm{Bloom}^{L}$, an extension to Bloom that takes inspiration from both of these traditions. Bloom ${ }^{L}$ generalizes Bloom to support lattices and extends the power of CALM analysis to whole programs containing arbitrary lattices. We show how the Bloom interpreter can be generalized to support efficient evaluation of lattice-based code using well-known strategies from logic programming. Finally, we use Bloom ${ }^{L}$ to develop several practical distributed programs, including a key-value store similar to Amazon Dynamo, and show how Bloom ${ }^{L}$ encourages the safe composition of small, easy-to-analyze lattices into larger programs.
\end{abstract}

\section{Categories and Subject Descriptors}

D.3.2 [Language Classifications]: Concurrent, distributed, and parallel languages

\section{General Terms}

Design, Languages

\section{Keywords}

Bloom, distributed programming, eventual consistency, lattice

\section{INTRODUCTION}

As cloud computing becomes increasingly common, the inherent difficulties of distributed programming - asynchrony, concurrency, and partial failure-affect a growing segment of the developer community. Traditionally, transactions and other forms of strong consistency encapsulated these problems at the data management layer. But

Permission to make digital or hard copies of all or part of this work for personal or classroom use is granted without fee provided that copies are not made or distributed for profit or commercial advantage and that copies bear this notice and the full citation on the first page. To copy otherwise, to republish, to post on servers or to redistribute to lists, requires prior specific permission and/or a fee.

SOCC'12, October 14-17, 2012, San Jose, CA USA

Copyright 2012 ACM 978-1-4503-1761-0/12/10 ...\$15.00. in recent years there has been interest in achieving application-level consistency criteria without incurring the latency and availability costs of strongly consistent storage $[8,19]$.

Two different frameworks for these techniques have received significant attention in recent research: Convergent Modules and Monotonic Logic.

Convergent Modules: In this approach, a programmer writes encapsulated modules whose public methods provide certain guarantees regarding message reordering and retry. For example, Statebox is an open-source library that merges conflicting updates to data items in a key-value store; the user of the library need only register "merge functions" that are commutative, associative, and idempotent [21]. This approach has roots in database and systems research $[14,16$, $19,29,41]$ as well as groupware [13, 39]. Shapiro et al. recently proposed a formalism for these approaches called Convergent Replicated Data Types (CvRDTs), which casts these ideas into the algebraic framework of semilattices [36, 37].

CvRDTs present two main problems: (a) the programmer bears responsibility for ensuring lattice properties for their methods (commutativity, associativity, idempotence), and (b) CvRDTs only provide guarantees for individual values, not for application logic in general. As an example of this second point, consider the following:

ExAmple 1. A replicated, fault-tolerant courseware application assigns students into study teams. It uses two set CvRDTs: one for Students and another for Teams. The application reads a version of Students and inserts the derived element $<$ Alice,Bob $>$ into Teams. Concurrently, Bob is removed from Students by another application replica. The use of CvRDTs ensures that all replicas will eventually agree that Bob is absent from Students, but this is not enough: application-level state is inconsistent unless the derived values in Teams are updated consistently to reflect Bob's removal. This is outside the scope of CvRDT guarantees.

Taken together, the problems with Convergent Modules present a scope dilemma: a small module (e.g., a set) makes lattice properties easy to inspect and test, but provides only simple semantic guarantees. Large CvRDTs (e.g., an eventually consistent shopping cart) provide higher-level application guarantees but require the programmer to ensure lattice properties hold for a complex module, resulting in software that is difficult to test, maintain, and trust.

Monotonic Logic: In recent work, we observed that the database theory literature on monotonic logic provides a powerful lens for reasoning about distributed consistency. Intuitively, a monotonic program makes forward progress over time: it never "retracts" an earlier conclusion in the face of new information. We proposed the CALM theorem, which established that all monotonic programs are confluent (invariant to message reordering and retry) and hence eventually consistent $[5,20,27]$. Monotonicity of a Datalog program 
is straightforward to determine conservatively from syntax, so the CALM theorem provides the basis for a simple analysis of the consistency of distributed programs. We concretized CALM into an analysis procedure for Bloom, a Datalog-based language for distributed programming [2,9].

The original formulation of CALM and Bloom only verified the consistency of programs that compute sets of facts that grow over time ("set monotonicity"); that is, "forward progress" was defined according to set containment. As a practical matter, this is overly conservative: it precludes the use of common monotonically increasing constructs such as timestamps and sequence numbers.

EXAMPLE 2. In a quorum voting service, a coordinator counts the number of votes received from participant nodes; quorum is reached once the number of votes exceeds a threshold. This is clearly monotonic: the vote counter increases monotonically, as does the threshold test (count(votes) > k) which "grows" from False to True. But both of these constructs (upward-moving mutable variables and aggregates) are labeled non-monotonic by the original CALM analysis.

The CALM theorem obviates any scoping concerns for convergent monotonic logic, but it presents a type dilemma. Sets are the only data type amenable to CALM analysis, but the programmer may have a more natural representation of a monotonically growing phenomenon. For example, a monotonic counter is more naturally represented as a growing integer than a growing set. This dilemma leads either to false negatives in CALM analysis and over-use of coordination, or to idiosyncratic set-based implementations that can be hard to read and maintain.

\subsection{Bloom $^{L}$ : Logic And Lattices}

We address the two dilemmas above with Bloom $^{L}$, an extension to Bloom that incorporates a semilattice construct similar to CvRDTs. We present this construct in detail below, but the intuition is that Bloom ${ }^{L}$ programs can be defined over arbitrary types - not just setsas long as they have commutative, associative, and idempotent merge functions ("least upper bound") for pairs of items. Such a merge function defines a partial order for its type. This generalizes Bloom (and traditional Datalog), which assumes a fixed merge function (set union) and partial order (set containment).

Bloom $^{L}$ provides three main improvements in the state of the art of both Bloom and CvRDTs:

1. Bloom ${ }^{L}$ solves the type dilemma of logic programming: CALM analysis in Bloom ${ }^{L}$ can assess monotonicity for arbitrary lattices, making it significantly more liberal in its ability to test for confluence. Bloom ${ }^{L}$ can validate the coordination-free use of common constructs like timestamps and sequence numbers.

2. Bloom ${ }^{L}$ solves the scope dilemma of CvRDTs by providing monotonicity-preserving mappings between lattices via morphisms and monotone functions. Using these mappings, the per-component monotonicity guarantees offered by CvRDTs can be extended across multiple items of lattice type. This capability is key to the CALM analysis described above. It is also useful for proving the monotonicity of sub-programs even when the whole program is not designed to be monotonic.

3. For efficient incremental execution, we extend the standard Datalog semi-naive evaluation scheme [7] to support lattices. We also describe how to extend an existing Datalog runtime to support lattices with relatively minor changes.

\subsection{Outline}

The remainder of the paper proceeds as follows. Section 2 provides background on Bloom and CALM. In Section 3 we introduce Bloom $^{L}$, including cross-lattice morphisms and monotone functions. We detail Bloom ${ }^{L}$ 's built-in lattice types and show how developers can define new lattices. We also describe how the CALM analysis extends to Bloom ${ }^{L}$. In Section 4, we describe how we modified the Bloom runtime to support Bloom ${ }^{L}$.

In Sections 5 and 6, we present two case studies. First, we use Bloom $^{L}$ to implement a distributed key-value store that supports eventual consistency, object versioning using vector clocks, and quorum replication. Second, we revisit the simple e-commerce scenario presented by Alvaro et al. in which clients interact with a replicated shopping cart service [2]. We show how Bloom ${ }^{L}$ can be used to make the "checkout" operation monotonic and confluent, despite the fact that it requires aggregation over a distributed data set.

\section{BACKGROUND}

In this section, we review the Bloom programming language and the CALM program analysis. We present a simple program for which the CALM analysis over sets yields unsatisfactory results.

\subsection{Bloom}

Bloom programs are bundles of declarative statements about collections of facts (tuples). An instance of a Bloom program performs computation by evaluating its statements over the contents of its local database. Instances communicate via asynchronous messaging.

An instance of a Bloom program proceeds through a series of timesteps, each containing three phases. ${ }^{1}$ In the first phase, inbound events (e.g., network messages) are received and represented as facts in collections. In the second phase, the program's statements are evaluated over local state to compute all the additional facts that can be derived from the current collection contents. In some cases (described below), a derived fact is intended to achieve a "side effect," such as modifying local state or sending a network message. These effects are deferred during the second phase of the timestep; the third phase is devoted to carrying them out.

The initial implementation of Bloom, called Bud, allows Bloom logic to be embedded inside a Ruby program. Figure 1 shows a Bloom program represented as an annotated Ruby class. A small amount of Ruby code is needed to instantiate the Bloom program and begin executing it; more details are available on the Bloom language website [9].

\subsubsection{Data Model}

The Bloom data model is based on collections. A collection is an unordered set of facts, akin to a relation in Datalog. The Bud prototype adopts the Ruby type system rather than inventing its own; hence, a fact in Bud is just an array of immutable Ruby objects. Each collection has a schema, which declares the structure (column names) of the facts in the collection. A subset of the columns in a collection form its key: as in the relational model, the key columns functionally determine the remaining columns. The collections used by a Bloom program are declared in a state block. For example, line 5 of Figure 1 declares a collection named link with three columns, two of which form the collection's key. Ruby is a dynamically typed language, so keys and values in Bud can hold arbitrary Ruby objects.

Bloom provides several collection types to represent different kinds of state (Table 1). A table stores persistent data: if a fact

\footnotetext{
${ }^{1}$ There is a declarative semantics for Bloom $[1,4]$, but for the sake of exposition we describe the language operationally here.
} 


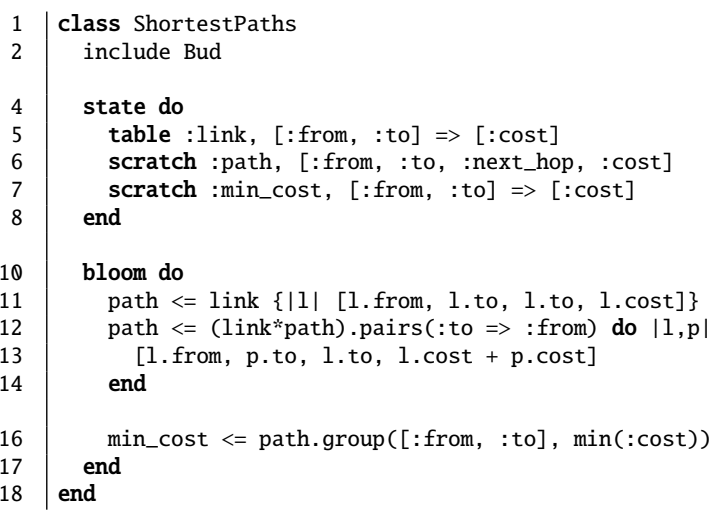

Figure 1: All-pairs shortest paths in Bloom.

appears in a table, it remains in the table in future timesteps (unless it is explicitly removed). A scratch contains transient data-the content of scratch collections is emptied at the start of each timestep. Scratches are akin to SQL views: they are often useful as a way to name intermediate results or as a "macro" construct to enable code reuse. A channel allows communication between Bloom instances. The schema of a channel has a distinguished location specifier column (prefixed with " $@$ "). When a fact is derived for a channel collection, it is sent to the remote Bloom instance at the address given by the location specifier.

\subsubsection{Statements}

Each Bloom statement has one or more input collections and a single output collection. A statement takes the form: $<$ collection-identifier $><$ op $><$ collection-expression $>$

The left-hand side (lhs) is the name of the output collection and the right-hand side (rhs) is an expression that produces a collection. A statement defines how the input collections are transformed before being included (via set union) in the output collection. Bloom allows the usual relational operators to be used on the rhs (selection, projection, join, grouping, aggregation, and negation), although it adopts a syntax intended to be more familiar to imperative programmers. In Figure 1, line 11 demonstrates projection, lines 12-14 perform a join between link and path using the join predicate link. to $=$ path. from followed by a projection to four attributes, and line 16 shows grouping and aggregation. Bloom statements appear in one or more bloom blocks, which group together semantically related statements to aid readability.

Bloom provides several operators that determine when the rhs will be merged into the lhs (Table 2 ). The $<=$ operator performs standard logical deduction: that is, the lhs and rhs are true at the same timestep. The $<+$ and $<-$ operators indicate that facts will be added to or removed from the lhs collection at the beginning of the next timestep. The $<\sim$ operator specifies that the rhs will be merged into the lhs collection at some non-deterministic future time. The lhs of a statement that uses $<\sim$ must be a channel; the $<\sim$ operator captures asynchronous messaging.

Bloom allows recursion-i.e., the rhs of a statement can reference the lhs collection, either directly or indirectly. As in Datalog, certain constraints must be adopted to ensure that programs with recursive statements have a sensible interpretation. For deductive statements ( $<=$ operator), we require that programs be syntactically stratified [6]: cycles through negation or aggregation are not allowed [4].

\begin{tabular}{|l|l|}
\hline Name & Behavior \\
\hline table & Persistent storage. \\
scratch & Transient storage. \\
& $\begin{array}{l}\text { Asynchronous communication. A fact derived } \\
\text { into a channel appears in the database of a } \\
\text { remote Bloom instance at a non-deterministic } \\
\text { future time. }\end{array}$ \\
\hline
\end{tabular}

Table 1: Bloom collection types.

\begin{tabular}{|c|l|l|}
\hline Op & Name & Meaning \\
\hline$<=$ & merge & $\begin{array}{l}\text { lhs includes the content of rhs in the } \\
\text { current timestep. }\end{array}$ \\
\hline$<+$ & deferred merge & $\begin{array}{l}\text { lhs will include the content of rhs in } \\
\text { the next timestep. }\end{array}$ \\
\hline$<-$ & deferred delete & $\begin{array}{l}\text { lhs will not include the content of rhs } \\
\text { in the next timestep. }\end{array}$ \\
\hline$<\sim$ & async merge & $\begin{array}{l}\text { (Remote) lhs will include the content } \\
\text { of the rhs at some non-deterministic } \\
\text { future timestep. }\end{array}$ \\
\hline
\end{tabular}

Table 2: Bloom operators.

\subsection{CALM Analysis}

Work on deductive databases has long drawn a distinction between monotonic and non-monotonic logic programs. Intuitively, a monotonic program only computes more information over time-it never "retracts" a previous conclusion in the face of new evidence. In Bloom (and Datalog), a simple conservative test for monotonicity is based on program syntax: selection, projection, and join are monotonic, while aggregation, negation, and deletion are not.

The CALM theorem connects the theory of monotonic logic with the practical problem of distributed consistency $[2,20]$. All monotonic programs are "eventually consistent" or confluent: for any given input, all program executions result in the same final state regardless of network non-determinism [5, 27]. Hence, monotonic logic is a useful building block for loosely consistent distributed programming.

According to the CALM theorem, distributed inconsistency may only occur at a point of order: a program location where an asynchronously derived value is consumed by a non-monotonic operator [2]. This is because asynchronous messaging results in nondeterministic arrival order, and non-monotonic operators may produce different conclusions when evaluated over different subsets of their inputs. For example, consider a Bloom program consisting of a pair of collections $A$ and $B$ (both fed by asynchronous channels) and a rule that sends a message whenever an element of $A$ arrives that is not in $B$. This program is non-monotonic and exhibits non-confluent behavior: the messages sent by the program will depend on the order in which the elements of $A$ and $B$ arrive.

We have implemented a conservative static program analysis in Bloom that follows directly from the CALM theorem. Programs that are free from non-monotonic constructs are "blessed" as confluent: producing the same output on different runs or converging to the same state on multiple distributed replicas. Otherwise, programs are flagged as potentially inconsistent. To achieve consistency, the programmer either needs to rewrite their program to avoid the use of non-monotonicity or introduce a coordination protocol to ensure that a consistent ordering is agreed upon at each of the program's points of order. Coordination protocols increase latency and reduce availability in the event of network partitions, so in this paper we focus on coordination-free designs- that is, monotonic programs. 


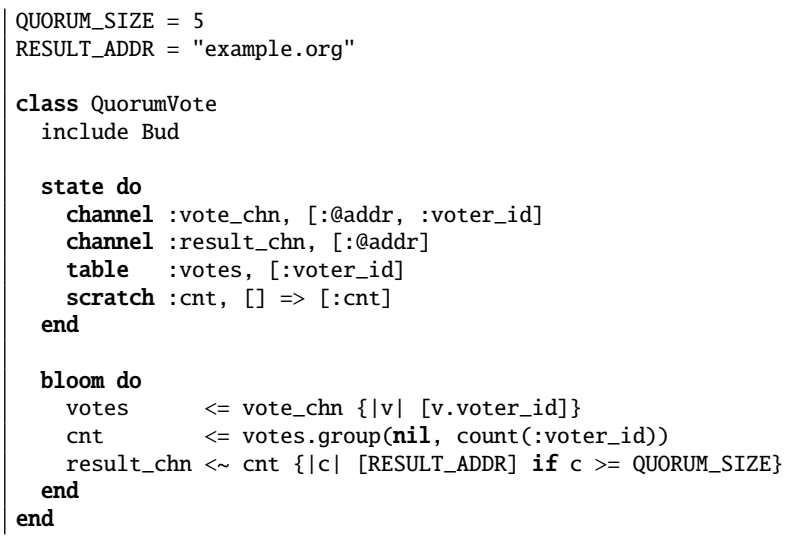

Figure 2: A non-monotonic Bloom program that waits for a quorum of votes to be received.

\subsubsection{Limitations Of Set Monotonicity}

The original formulation of the CALM theorem considered only programs that compute more facts over time-that is, programs whose sets grow monotonically. Many distributed protocols make progress over time but their notion of "progress" is often difficult to represent as a growing set of facts. For example, consider the Bloom program in Figure 2. This program receives votes from one or more clients (not shown) via the vote_chn channel. Once at least QUORUM_SIZE votes have been received, a message is sent to a remote node to indicate that quorum has been reached (line 17). This program resembles a "quorum vote" subroutine that might be used by an implementation of Paxos [24] or quorum replication [18].

Intuitively, this program makes progress in a semantically monotonic fashion: the set of received votes grows and the size of the votes collection can only increase, so once a quorum has been reached it will never be retracted. Unfortunately, the current CALM analysis would regard this program as non-monotonic because it contains a point of order: the grouping operation on line 16 .

To solve this problem, we need to introduce a notion of program values that "grow" according to a partial order other than set containment. We do this by extending Bloom to operate over arbitrary lattices rather than just the set lattice.

\section{ADDING LATTICES TO BLOOM}

This section introduces Bloom ${ }^{L}$, an extension to Bloom that allows monotonic programs to be written using arbitrary lattices. We begin by reviewing the algebraic properties of lattices, monotone functions, and morphisms. We then introduce the basic concepts of Bloom $^{L}$ and detail the built-in lattices provided by the language. We also show how users can define their own lattice types.

When designing Bloom ${ }^{L}$, we decided to extend Bloom to include support for lattices rather than building a new language from scratch. Hence, Bloom ${ }^{L}$ is backward compatible with Bloom and was implemented with relatively minor changes to the Bud runtime. We describe how code written using lattices can interoperate with traditional Bloom collections in Section 3.5.

\subsection{Definitions}

A bounded join semilattice is a triple $\langle S, \sqcup, \perp\rangle$, where $S$ is a set, $\sqcup$ is a binary operator (called "join" or "least upper bound"), and $\perp$ is an element of $S$ (called "bottom"). The $\sqcup$ operator is associative, commutative, and idempotent. The $\sqcup$ operator induces a partial order

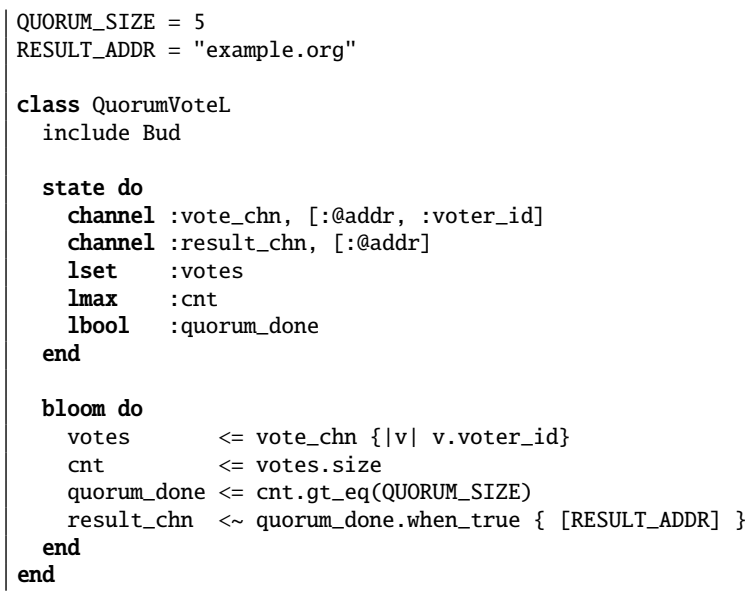

Figure 3: A monotonic Bloom ${ }^{L}$ program that waits for a quorum of votes to be received.

$\leq_{S}$ on the elements of $S: x \leq_{S} y$ if $x \sqcup y=y$. Note that although $\leq_{S}$ is only a partial order, the least upper bound is defined for all elements $x, y \in S$. The distinguished element $\perp$ is the smallest element in $S$ : $x \sqcup \perp=x$ for every $x \in S$. For brevity, we use the term "lattice" to mean "bounded join semilattice" in the rest of this paper. We use the informal term "merge function" to mean "least upper bound."

A monotone function is a function $f: S \rightarrow T$ such that $S, T$ are partially ordered sets (posets) and $\forall a, b \in S: a \leq_{S} b \Rightarrow f(a) \leq_{T}$ $f(b)$. That is, $f$ maps elements of $S$ to elements of $T$ in a manner that respects the partial orders of both posets. ${ }^{2}$

A morphism from lattice $\left\langle X, \sqcup_{X}, \perp_{X}\right\rangle$ to lattice $\left\langle Y, \sqcup_{Y}, \perp_{Y}\right\rangle$ is a function $g: X \rightarrow Y$ such that $g\left(\perp_{X}\right)=\perp_{Y}$ and $\forall a, b \in X: g\left(a \sqcup_{X}\right.$ $b)=g(a) \sqcup_{Y} g(b)$. Intuitively, $g$ maps elements of $X$ to elements of $Y$ in a way that preserves the lattice properties. Note that morphisms are monotone functions but the converse is not true in general.

\subsection{Language Constructs}

Bloom $^{L}$ allows both lattices and collections to represent state. A lattice is analogous to a collection type in Bloom, while a lattice element corresponds to a particular collection. For example, the lset lattice is similar to the table collection type provided by Bloom; an element of the lset lattice is a particular set. In the terminology of object-oriented programming, a lattice is a class that obeys a certain interface and an element of a lattice is an instance of that class. Figure 3 contains an example Bloom ${ }^{L}$ program.

As with collections, the lattices used by a Bloom ${ }^{L}$ program are declared in a state block. More precisely, a state block declaration introduces an identifier that is associated with a lattice element; over time, the binding between identifiers and lattice elements is updated to reflect state changes in the program. For example, line 10 of Figure 3 declares an identifier votes that is mapped to an element of the lset lattice. As more votes are received, the lattice element associated with the votes identifier changes (it moves "upward" in the lset lattice). When a lattice identifier is declared, it is initially bound to $\perp$, the smallest element in the lattice. For example, an lset lattice initially contains the empty set.

\footnotetext{
${ }^{2}$ To simplify the presentation, these definitions assume that monotone functions and morphisms are unary. Bloom ${ }^{L}$ supports monotone functions and morphisms with any number of arguments.
} 


\begin{tabular}{|c|c|c|c|c|c|}
\hline Name & Description & Least element $(\perp)$ & $\operatorname{Merge}(a, b)$ & Morphisms & Monotone functions \\
\hline lbool & $\begin{array}{l}\text { Boolean lattice } \\
\text { (false } \rightarrow \text { true) }\end{array}$ & false & $a \vee b$ & when_true $(\& b l k) \rightarrow v$ & \\
\hline $\operatorname{lmax}$ & $\begin{array}{l}\text { Max over an } \\
\text { ordered domain }\end{array}$ & $-\infty$ & $\max (a, b)$ & $\begin{array}{l}\text { gt }(n) \rightarrow 1 \text { bool } \\
\text { gt_eq }(n) \rightarrow 1 \text { bool } \\
+(n) \rightarrow 1 \max \\
-(n) \rightarrow 1 \max \end{array}$ & \\
\hline $\operatorname{lmin}$ & $\begin{array}{l}\text { Min over an } \\
\text { ordered domain }\end{array}$ & $\infty$ & $\min (a, b)$ & $\begin{array}{l}\text { lt }(n) \rightarrow 1 \text { bool } \\
\text { lt_eq }(n) \rightarrow 1 \text { bool } \\
+(n) \rightarrow \operatorname{lmin} \\
-(n) \rightarrow \operatorname{lmin}\end{array}$ & \\
\hline lset & Set of values & empty set & $a \cup b$ & $\begin{array}{l}\text { intersect }(\text { lset }) \rightarrow \text { lset } \\
\text { project }(\& b l \mathrm{k}) \rightarrow 1 \text { set } \\
\text { product }(l \text { set }) \rightarrow 1 \text { set } \\
\text { contains? }(v) \rightarrow 1 \text { bool }\end{array}$ & $\operatorname{size}() \rightarrow \operatorname{lmax}$ \\
\hline lpset & $\begin{array}{l}\text { Set of non- } \\
\text { negative numbers }\end{array}$ & empty set & $a \cup b$ & $\begin{array}{l}\text { intersect }(\text { lpset }) \rightarrow \text { lpset } \\
\text { project }(\& b l k) \rightarrow \text { lpset } \\
\text { product }(l \text { pset }) \rightarrow \text { lpset } \\
\text { contains? }(v) \rightarrow \text { lbool }\end{array}$ & $\begin{array}{l}\operatorname{size}() \rightarrow 1 \max \\
\operatorname{sum}() \rightarrow 1 \max \end{array}$ \\
\hline lbag & Multiset of values & empty multiset & $a \cup b$ & $\begin{array}{l}\text { intersect(lbag) } \rightarrow \text { lbag } \\
\text { project }(\& b l k) \rightarrow \text { lbag } \\
\text { multiplicity }(v) \rightarrow \text { lmax } \\
\text { contains? (v) } \rightarrow \text { lbool } \\
+(\text { lbag }) \rightarrow \text { lbag }\end{array}$ & $\operatorname{size}() \rightarrow \operatorname{lmax}$ \\
\hline lmap & $\begin{array}{l}\text { Map from keys to } \\
\text { lattice values }\end{array}$ & empty map & see text & $\begin{array}{l}\text { intersect (lmap) } \rightarrow \text { lmap } \\
\text { project }(\& b l \mathrm{k}) \rightarrow \text { lmap } \\
\text { key_set }() \rightarrow \text { lset } \\
\text { at }(\mathrm{v}) \rightarrow \text { any-lattice } \\
\text { key? }(\mathrm{v}) \rightarrow \text { lbool }\end{array}$ & $\operatorname{size}() \rightarrow \operatorname{lmax}$ \\
\hline
\end{tabular}

Table 3: Built-in lattices in Bloom ${ }^{L}$. Note that $v$ denotes a Ruby value, $n$ denotes a number, and blk indicates a Ruby code block (anonymous function).

\subsubsection{Statements}

Statements take the same form in both Bloom and Bloom ${ }^{L}$ :

$<$ identifier $><o p><$ expression $>$

The identifier on the lhs can refer to either a set-oriented collection or a lattice element. The expression on the rhs can contain both traditional relational operators (applied to Bloom collections) and methods invoked on lattices. Lattice methods are similar to methods in an object-oriented language and are invoked using the standard Ruby method invocation syntax. For example, line 17 of Figure 3 invokes the size method on an element of the lset lattice.

If the lhs is a lattice, the statement's operator must be either $<=$ or $<+$ (instantaneous or deferred deduction, respectively). The meaning of these operators is that, at either the current or the following timestep, the lhs identifier will take on the result of applying the lattice's least upper bound to the lhs and rhs lattice elements. The intuition remains the same as in Bloom: the rhs value is "merged into" the lhs lattice, except that the semantics of the merge operation are defined by the lattice's least upper bound operator. We require that the lhs and rhs refer to a lattice of the same type.

Bloom $^{L}$ does not support deletion (<- operator) for lattices. Lattices do not directly support asynchronous communication (via the $<\sim$ operator) but lattice elements can be embedded into facts that appear in channels (Section 3.5.2).

\subsubsection{Lattice Methods}

Bloom $^{L}$ statements compute values over lattices by invoking methods on lattice elements. Just as a subset of the relational algebra is monotonic, some lattice methods are monotone functions (as defined in Section 3.1). A monotone lattice method guarantees that, if the lattice on which the method is invoked grows (according to the lattice's partial order), the value returned by the method will grow (according to the return value's lattice type). For example, the size method provided by the lset lattice is monotone because as more elements are added to the set, the size of the set increases. Intuitively, a lattice's monotone methods constitute a "safe" interface of operations that can be invoked in a distributed setting without risk of inconsistency.

A lattice method's signature indicates its monotonicity properties. Bloom $^{L}$ distinguishes between methods that are monotone and a subset of monotone methods that are morphisms. Section 3.1 defines the properties that a morphism must satisfy, but the intuition is that a morphism on lattice $T$ can be distributed over $T$ 's least upper bound. For example, the size method of the lset lattice is not a morphism. To see why, consider two elements of the lset lattice, $\{1,2\}$ and $\{2,3\}$. The size method is not a morphism because $\operatorname{size}\left(\{1,2\} \sqcup_{\text {lset }}\{2,3\}\right) \neq \operatorname{size}(\{1,2\}) \sqcup_{1 \max } \operatorname{size}(\{2,3\})$. Morphisms can be evaluated more efficiently than monotone methods, as we discuss in Section 4.1.

Lattices can also define non-monotonic methods. Using a nonmonotonic lattice method is analogous to using a non-monotonic relational operator in Bloom: the Bud interpreter stratifies the program to ensure that the input value is computed to completion before allowing the non-monotonic method to be invoked. Bloom ${ }^{L}$ encourages developers to minimize the use of non-monotonic constructs: as the CALM analysis suggests, non-monotonic reasoning may need to be augmented with coordination to ensure consistent results.

Every lattice defines a non-monotonic reveal method that returns a representation of the lattice element as a plain Ruby value. For example, the reveal method on an Imax lattice returns a Ruby integer. This method is non-monotonic because once the underlying Ruby value has been extracted from the lattice, Bloom $^{L}$ cannot ensure that subsequent code uses the value in a monotonic fashion. 


\subsection{Built-in Lattices}

Table 3 lists the lattices included with Bloom ${ }^{L}$. The built-in lattices provide support for several common notions of "progress": a predicate that moves from false to true (lbool), a numeric value that strictly increases or strictly decreases (lmax and lmin, respectively), and various kinds of collections that grow over time (lset, lpset, lbag, and lmap). The behavior of most of the lattice methods should be unsurprising, so we do not describe every method in this section.

The lbool lattice represents conditions that, once satisfied, remain satisfied. For example, the gt morphism on the lmax lattice takes a numeric argument $n$ and returns an 1bool; once the lmax exceeds $n$, it will remain $>n$. The when_true morphism takes a Ruby block; if the lbool element has the value true, when_true returns the result of evaluating the block. For example, see line 19 in Figure 3. when_true is similar to an "if" statement. ${ }^{3}$

The collection-like lattices support familiar operations such as union, intersection, and testing for the presence of an element in the collection. The project morphism takes a code block and forms a new collection by applying the code block to each element of the input collection. Elements for which the code block returns nil are omitted from the output collection, which allows project to be used as a filter.

The lbag lattice demonstrates how Bloom ${ }^{L}$ can support multisets. Note that the lbag merge function takes the maximum of the multiplicities of an element that appears in both input lattices. Although this is the standard definition of multiset union, some applications might find summing the element multiplicities to be more useful. However, this behavior would not be a valid least upper bound (because it is not idempotent). Instead, applications that need to compute the multiset sum can use the + morphism.

The lpset lattice is an example of how Bloom ${ }^{L}$ can be used to encode domain-specific knowledge about an application. If the developer knows that a set will only contain non-negative numbers, the sum of those numbers increases monotonically as the set grows. Hence, sum is a monotone function of lpset; in contrast, taking the sum of the elements of an lset is non-monotonic in general.

The lmap lattice associates keys with values. Keys are immutable Ruby objects and values are lattice elements. For example, a web application could use an lmap to associate session IDs with an lset containing the pages visited by that session. The lmap merge function takes the union of the key sets of its input maps. If a key occurs in both inputs, the two corresponding values are merged using the appropriate lattice merge function. Note that the at (v) morphism returns the lattice element associated with key $\mathrm{v}$ (or $\perp$ if the lmap does not contain $\mathrm{v}$ ).

\subsection{User-defined Lattices}

The built-in lattices are sufficient to express many programs. However, Bloom ${ }^{L}$ also allows developers to create custom lattices to capture domain-specific behavior. To define a new lattice, a developer creates a Ruby class that meets a certain API contract. Figure 4 shows a simple implementation of the lset lattice using a Ruby array for storage. ${ }^{4}$

A lattice class must inherit from the built-in Bud: : Lattice class and must also define two methods:

- initialize(i): given a Ruby object $i$, this method constructs a new lattice element that "wraps" $i$ (this is the stan-

\footnotetext{
${ }^{3}$ Observe that an "else" clause would test for an upper bound on the final lattice value, which is a non-monotonic property.

${ }^{4} \mathrm{We}$ omit a few lset methods for brevity. While we use an array for simplicity, this is inefficient (e.g., duplicate elimination is expensive). The built-in lset implementation uses a hash-based set data type.
}

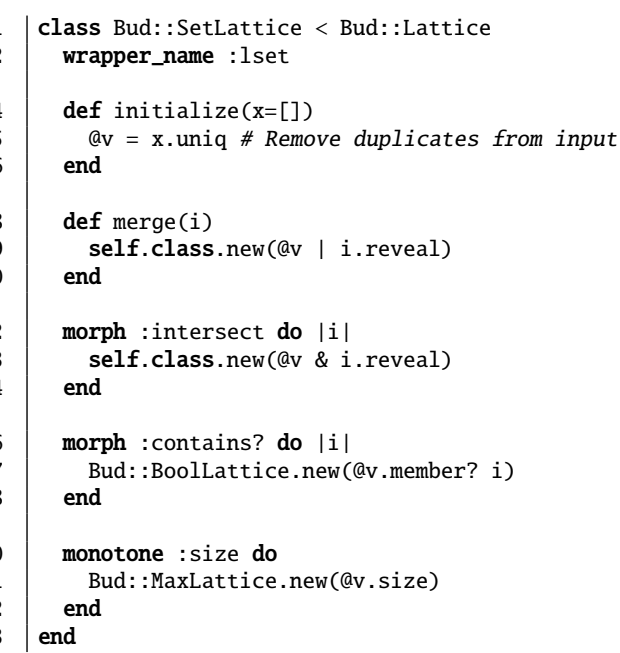

Figure 4: Example implementation of the lset lattice.

dard Ruby syntax for defining a constructor). If $i$ is nil (the null reference), this method returns $\perp$.

- merge (e): given a lattice element $e$, this method returns the least upper bound of $e$ and self. The programmer must ensure that this method satisfies the algebraic properties of least upper bound-in particular, it must be commutative, associative, and idempotent (Section 3.1). Note that $e$ and self must be instances of the same class.

Lattices can also define any number of monotone functions, morphisms, and non-monotonic methods. The syntax for declaring morphisms and monotone functions can be seen in lines 12-14 and 20-22 of Figure 4, respectively. Note that lattice elements are immutable - that is, lattice methods (including merge methods) must return new values, rather than modifying any of their inputs.

A lattice definition must specify a keyword that can be used in Bloom $^{L}$ state blocks. This is done using the wrapper_name class method. For example, line 2 of Figure 4 means that "lset : foo" in a state block will introduce an identifier foo that is associated with an instance of Bud: : SetLattice.

\subsection{Integration With Set-oriented Logic}

Bloom $^{L}$ provides two features to ease integration of lattice-based code with Bloom rules that use set-oriented collections.

\subsubsection{Converting Collections Into Lattices}

This feature enables an intuitive syntax for merging the contents of a set-oriented collection into a lattice. If a statement has a Bloom collection on the rhs and a lattice on the lhs, the collection is converted into a lattice element by "folding" the lattice's merge function over the collection. That is, each element of the collection is converted to a lattice element (by invoking the lattice constructor) and then the resulting lattice elements are merged together via repeated application of the lattice's merge method. In our experience, this is usually the behavior intended by the user.

For example, line 16 of Figure 3 contains a Bloom collection on the rhs and an lset lattice on the lhs. This statement is evaluated by constructing a singleton lset for each fact in the rhs collection and then merging the sets together. The resulting lset is then merged with the votes lattice referenced by the lhs. 


\subsubsection{Embedding Lattice Values In Collections}

Bloom $^{L}$ allows lattice elements to be used as column values of facts in Bloom collections. This feature allows Bloom ${ }^{L}$ programs to use a mixture of Bloom-style relational operators and lattice method invocations, depending on which is more convenient. Bloom also provides several collection types with special semantics (e.g., network communication, durable storage); allowing lattice elements to be embedded into collections avoids the need to create a redundant set of facilities for lattices.

Consider a simple Bloom ${ }^{L}$ statement that derives facts with an embedded lattice element as a column:

$$
\mathrm{t} 1<=\mathrm{t} 2\{|\mathrm{t}|[\mathrm{t} . \mathrm{x}, \mathrm{cnt}]\}
$$

where $t 1$ and $t 2$ are collections, cnt is a lattice, and the key of $t 1$ is its first column. Note that cnt might change over the course of a single timestep (specifically, cnt can increase according to the lattice's partial order). This might result in deriving multiple $t 1$ facts that differ only in the second column, which would violate $t 1$ 's key.

To resolve this situation, Bloom ${ }^{L}$ allows multiple facts to be derived that differ only in their embedded lattice values; those facts are merged into a single fact using the lattice's merge function. This is similar to specifying a procedure for how to resolve key constraint violations, a feature supported by some databases [30, 38]. For similar reasons, lattice elements cannot be used as keys in Bloom collections.

\subsection{CALM Analysis For Lattices}

As discussed in Section 2.2, CALM-based program analysis determines whether a program is order-sensitive by looking for "points of order"-locations where an asynchronously computed value is consumed by a non-monotonic operator. Bloom ${ }^{L}$ simply expands the set of monotonic operations that a program can contain; hence, we did not need to make any fundamental changes to our program analysis. Instead, we simply replaced the hard-coded list of monotonic operations with a list of the monotonic methods defined by the lattices used by the current program. In Sections 5 and 6, we show how CALM analysis can be applied to several Bloom ${ }^{L}$ programs.

CALM analysis is grounded in a model-theoretic characterization of confluence [27]. This work was done in the context of Dedalus, the formal language on which Bloom is based [4]. These results apply directly to Bloom, whose semantics are grounded in those of Dedalus. Monotonicity analysis in Bloom ${ }^{L}$ is a natural extension of the work on Bloom, but the development of a formal model-theoretic semantics for Bloom ${ }^{L}$ remains a topic for future work.

\section{IMPLEMENTATION}

In this section, we describe how to evaluate $B^{\prime} \operatorname{loom}^{L}$ programs. First, we generalize semi-naive evaluation to support lattices. We validate that our implementation of semi-naive evaluation results in significant performance gains and is competitive with the traditional set-only semi-naive evaluation scheme in Bud. We also describe how we extended Bud to support Bloom ${ }^{L}$ with relatively few changes.

\subsection{Evaluation Strategy}

Naive evaluation is a simple but inefficient approach to evaluating recursive Datalog programs. Evaluation proceeds in rounds. In each round, every rule in the program is evaluated over the entire database, including all derivations made in previous rounds. This process stops when a round makes no new derivations. Naive evaluation is inefficient because it makes many redundant derivations: once a fact has been derived in round $i$, it is rederived in every round $>i$.

Semi-naive evaluation improves upon naive evaluation by making fewer redundant derivations [7]. Let $\Delta_{0}$ represent the initial database

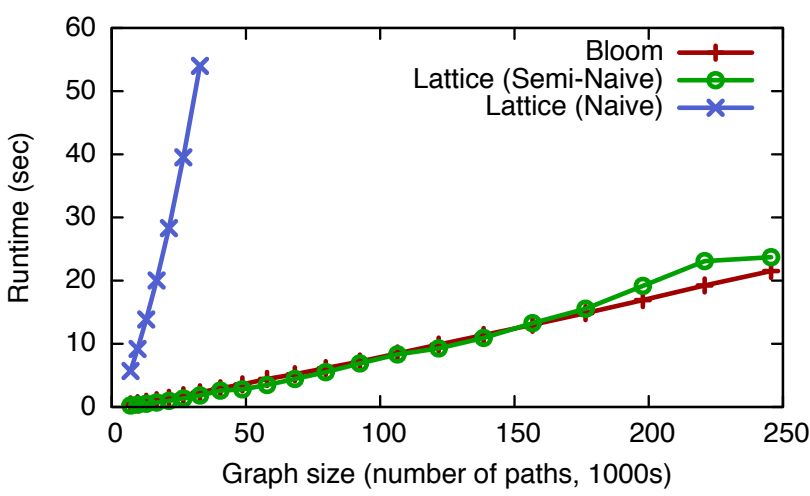

Figure 5: Performance of three different methods for computing the transitive closure of a graph.

state. In the first round, all the rules are evaluated over $\Delta_{0}$; let $\Delta_{1}$ represent the new facts derived in this round. In the second round, we only need to compute derivations that depend on $\Delta_{1}$ because everything that can be derived purely from $\Delta_{0}$ has already been computed.

A similar evaluation strategy works for Bloom ${ }^{L}$ statements that use lattice morphisms. For a given lattice identifier $l$, let $\Delta_{l}^{0}$ represent the lattice element associated with $l$ at the start of the current timestep. Let $\Delta_{l}^{r}$ represent the new derivations for $l$ that have been made in evaluation round $r$. During round one, the program's statements are evaluated and $l$ is mapped to $\Delta_{l}^{0}$; this computes $\Delta_{l}^{1}$. In round two, $l$ is now mapped to $\Delta_{l}^{1}$ and evaluating the program's statements yields $\Delta_{l}^{2}$. This process continues until $\Delta_{l}^{i}=\Delta_{l}^{i+1}$ for all identifiers $l$. The final value for $l$ is given by $\bigsqcup_{l: j=0}^{i} \Delta_{l}^{j}$.

This optimization cannot be used for monotone functions that are not morphisms. This is because semi-naive evaluation requires that we apply functions to the partial results derived in each round $k$ into $\Delta_{l}^{k}$, and later combine them using the lattice's merge operationeffectively distributing the function across the merge. For example, consider computing the lset lattice's size method, which returns an Imax lattice. The semi-naive strategy would compute $\bigsqcup_{\text {lmax: } j=0}^{i} \operatorname{size}\left(\Delta_{\text {lset }}^{j}\right)$ - the maximum of the sizes of the incremental results produced in each round. Thus it produces a different result than naive evaluation, which evaluates the size function against the complete database state in each round.

Implementing semi-naive style evaluation for lattices was straightforward. For each lattice identifier $l$, we record two values: a "total" value (the least upper bound of the derivations made for $l$ in all previous rounds) and a "delta" value (the least upper bound of the derivations made for $l$ in the last round). We implemented a program rewrite that examines each Bloom ${ }^{L}$ statement. If a statement only applies morphisms to lattice elements, the rewrite adjusts the statement to use the lattice's delta value rather than its total value.

\subsection{Performance Validation}

To validate the effectiveness of semi-naive evaluation for Bloom ${ }^{L}$ programs, we wrote two versions of a program to compute the transitive closure of a directed acyclic graph. One version was written in Bloom and used Bloom collections. The other version was written in Bloom ${ }^{L}$ using morphisms over the lset lattice. For the Bloom ${ }^{L}$ version, we ran the program both with and without semi-naive evaluation enabled. As input, we generated synthetic graphs of various sizes - in a graph with $n$ nodes, each node had roughly $\log _{2} n$ outgoing edges. We ran the experiment using a $2.13 \mathrm{GHz}$ Intel Core 2 
Duo processor and 4GB of RAM, running Mac OS X 10.7.4 and Ruby 1.9.3-p194. We ran each program variant five times on each graph and report the mean elapsed wall-clock time.

Figure 5 shows how the runtime of each program varied with the size of the graph. Note that we only report results for the naive Bloom $^{L}$ strategy on small input sizes because this variant ran very slowly as the graph size increased. The poor performance of naive evaluation is not surprising: after deriving all paths of length $n$, naive evaluation will then rederive all those paths at every subsequent round of the fixpoint computation. In contrast, after computing length $n$ paths, a semi-naive strategy will only generate length $n+1$ paths in the next round. Bloom and semi-naive Bloom ${ }^{L}$ achieve similar results. We instrumented Bud to count the number of derivations made by the Bloom and semi-naive lattice variants-as expected, both programs made a similar number of derivations. These results suggest that our implementation of semi-naive evaluation for Bloom $^{L}$ is effective and performs comparably with a traditional implementation of semi-naive evaluation for sets.

For large inputs, Bloom began to outperform the semi-naive lattice variant. We suspect this is because the lattice implementation copies more data than Bloom does for this benchmark. Lattice elements are immutable, so the lset merge function allocates a new object to hold the result of the merge. In contrast, Bloom collections are modified in-place. We plan to improve the lattice runtime to avoid copies when it can determine that in-place updates are safe.

\subsection{Discussion}

When designing Bloom ${ }^{L}$, we chose to extend the original Bloom language rather than inventing a new language from scratch. This design philosophy also applied to our language implementation: we found we were able to to extend Bud to support Bloom ${ }^{L}$ with relatively minor changes.

Bud initially had about 7300 lines of Ruby source code (LOC). Adding support for Bloom ${ }^{L}$ required less than 1000 lines of new or modified code; moreover, most of these changes were cleanly separated from the core Bud code. For example, the built-in lattice types constituted 300 LOC and support for lattice-based query plan elements required 250 LOC. In contrast, we had to make no changes to Bud's core fixpoint loop and extending CALM analysis to support lattices required modifying only 10 LOC. This experience suggests that support for lattices can be added to an existing Datalog engine in a relatively straightforward manner.

\section{CASE STUDY: KEY-VALUE STORE}

The next two sections contain case studies that show how Bloom ${ }^{L}$ can be used to build practical distributed programs. ${ }^{5}$ Both case studies are monotonic: that is, both programs consist of monotone functions applied to lattices. As a result, the Bloom ${ }^{L}$ compiler can verify that both of them are eventually consistent.

In the first case study, we show that a distributed, eventually consistent key-value store can be composed via a series of monotonic mappings between simple lattices. This example shows how Bloom ${ }^{L}$ overcomes the "scope dilemma" of CvRDTs: by composing a complex program from simple lattices (mostly Bloom ${ }^{L}$ built-ins), we can feel confident that individual lattices are correct, while CALM analysis finishes the job of verifying whole-program consistency.

\subsection{Basic Architecture}

A key-value store (KVS) provides a lookup service that allows client applications to store and retrieve the value associated with a given key. Key-value pairs are typically replicated on multiple

\footnotetext{
${ }^{5}$ Complete code listings for these case studies are available at
} http://db.cs. berkeley.edu/bloom-lattice.

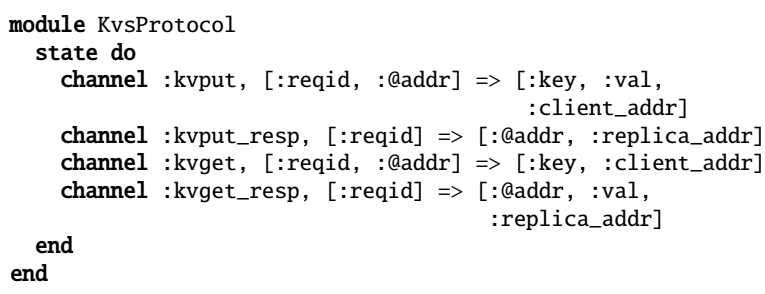

Figure 6: Key-value store interface.

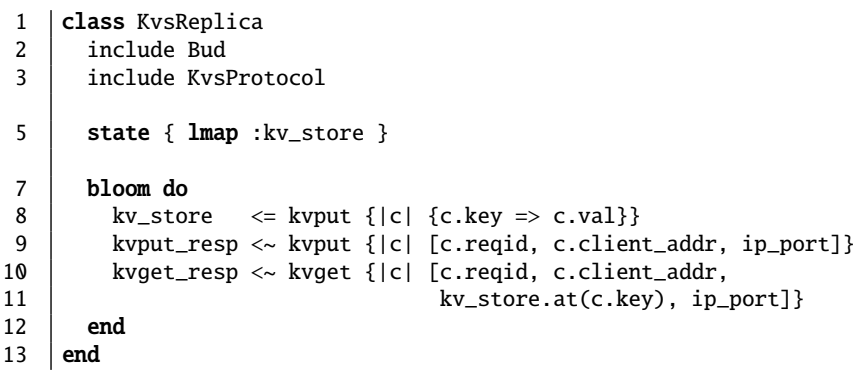

Figure 7: KVS replica implementation in Bloom $^{L}$.

storage nodes for redundancy and the key space is partitioned to improve aggregate storage and throughput. Eventual consistency is a common correctness criterion: after all client updates have reached all storage nodes, all the replicas of a key-value pair will converge to the same final state $[41,43]$.

Figure 6 shows a simple KVS interface in Bloom ${ }^{L}$. Client applications initiate get(key) and put(key, val) operations by inserting facts into the kvget and kvput channels, respectively; server replicas return responses via the kvget_resp and kvput_resp channels.

Figure 7 contains the Bloom ${ }^{L}$ code for a KVS server replica. An lmap lattice is used to maintain the mapping between keys and values (line 5). Since the values in an lmap lattice must themselves be lattice elements, for now we assume that clients only want to store and retrieve monotonically increasing lattice values; we discuss how to lift this restriction in Section 5.2. To handle a put(key, val) request, a new key $\rightarrow$ val lmap is created and merged into kv_store (line 8). If kv_store already contains a value for the given key, the two values will be merged together using the value lattice's merge function (see Section 3.3 for details). Observe that we use the Bloom ${ }^{L}$ features described in Section 3.5 to allow traditional Bloom collections (e.g., channels) and lattices (e.g., the kv_store lattice) to be used by the same program. Note also that ip_port is a built-in function that returns the IP address and port number of the current Bud instance.

The state of two replicas can be synchronized by simply exchanging their kv_store maps; the lmap merge function automatically resolves conflicting updates made to the same key. This property allows considerable flexibility in how replicas propagate updates.

\subsection{Object Versioning}

The basic KVS design is sufficient for applications that want to store monotonically increasing values such as session logs or increasing counters. To allow storage of values that change in arbitrary ways, we now consider how to support object versions. This is a classic technique for recognizing and resolving mutual inconsistency between members of a distributed system [32]. Our design is similar to that used by Amazon Dynamo [12]. 


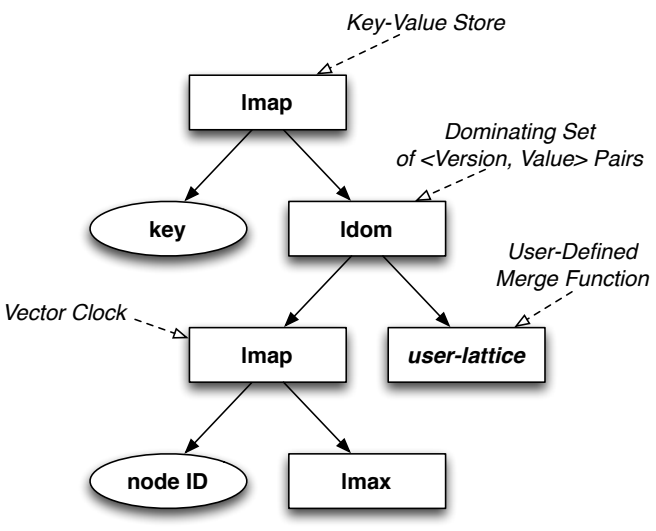

Figure 8: Lattice structure of a KVS with object versioning. Rectangles are lattices and ovals are atomic values.

Each replica associates keys with sets of $\langle$ vector-clock, value $\rangle$ pairs. The vector clock captures the causal relationship between different versions of a record [15, 28]. Clients store and retrieve $\langle$ vector-clock, value $\rangle$ pairs. When a client updates a value it has previously read, the client increments its own position in the vector clock and includes the updated vector clock $V_{U}$ with its put operation. Upon receiving an update, the server compares $V_{U}$ with the set of vector clocks associated with the updated key. For each such vector clock $V_{S}$, the server considers three cases:

1. $V_{U}>V_{S}$ : the client's update happens-after $V_{S}$, so $V_{S}$ is replaced with $V_{U}$.

2. $V_{U}<V_{S}$ : the client's update happens-before $V_{S}$, so $V_{S}$ is retained. ${ }^{6}$

3. $V_{U}$ and $V_{S}$ are incomparable: the two versions are concurrent, so both $V_{U}$ and $V_{S}$ are retained.

By applying these rules, each replica retains a set of mutually concurrent versions for each key. To handle a get operation, the server returns a single $\langle$ vector-clock, value $\rangle$ pair by merging together the vector clocks and using a user-supplied reconciliation function to merge together the conflicting values, as described below. We call the set of incomparable version-value pairs a dominating set.

From a Bloom ${ }^{L}$ perspective, each replica still stores a monotonically increasing value - the only difference is that in this scheme, the version stored by a replica increases over time, rather than the associated value. Hence, we now consider how to support vector clocks and dominating sets using Bloom $^{L}$.

\subsubsection{Vector Clocks}

A vector clock is a map from node identifiers to logical clocks $[15$, 28]. Let $V_{e}$ denote the vector clock for event $e$; let $V_{e}(n)$ denote the logical clock associated with node $n$ in $V_{e}$. If $V_{e}<V_{e^{\prime}}, e$ causally precedes $e^{\prime}$, where

$$
V_{e}<V_{e^{\prime}} \equiv \forall x\left[V_{e}(x) \leq V_{e^{\prime}}(x)\right] \wedge \exists y\left[V_{e}(y)<V_{e^{\prime}}(y)\right]
$$

In Bloom ${ }^{L}$, a vector clock can be represented as an lmap that maps node identifiers to Imax values. Each Imax represents the logical clock of a single node; this is appropriate because the logical clock value associated with a given node will only increase over time. The merge function provided by lmap achieves the desired semantics-

${ }^{6}$ This situation might arise due to duplication and reordering of messages by the network. that is, the built-in least upper bound for an lmap that contains Imax values is consistent with the partial order given above. ${ }^{7}$

\subsubsection{Dominating Sets}

We use a custom lattice type ldom to represent a dominating set. As discussed above, a dominating set is a set of 〈version,value $\rangle$ pairs; both elements of the pair are themselves represented as lattice values. In the KVS, the version lattice is a vector clock (that is, an lmap containing lmax values); the value lattice is whatever value the user wants to store in the KVS. ${ }^{8}$

The discussion above suggests a natural merge function for ldom: given two input ldom elements $e$ and $e^{\prime}$, the merge function omits all "dominated" pairs. For example, a $\langle$ version, value $\rangle$ pair in $e$ is included in the result of the merge unless there is a pair in $e^{\prime}$ whose version is strictly greater.

The ldom lattice provides two functions, version and value, that return the least upper bound of the concurrent versions or values, respectively. In the KVS, the value function corresponds to reconciling conflicting updates, whereas version returns the vector clock associated with the reconciled value. Note that while the version of a given ldom increases over time (as new versions are observed), the value does not; hence, version is a monotone function but value is not. Since the goal of object versioning is to allow values to change in non-monotonic ways, this is the expected behavior.

\subsubsection{Discussion}

Figure 8 shows the lattices used in a KVS with object versioning. Surprisingly, adding support for object versioning did not require any changes to the KVS replica code. Instead, clients simply store ldom values containing a single $\langle$ vector-clock, value $\rangle$ pair, incrementing their position in the vector clock as described above. The KVS replica merges these ldom values into an lmap as usual; the ldom merge function handles conflict resolution in the appropriate manner. Moreover, by composing the KVS from a collection of simple lattices, we found it easy to reason about the behavior of the system. For example, convincing ourselves that KVS replicas will eventually converge only required checking that the individual ldom, Imap, and lmax lattices satisfy the lattice properties, rather than analyzing the behavior of the system as a whole.

Our design compares favorably to traditional implementations of object versioning and vector clocks. For example, the implementation of vector clocks alone in the Voldemort KVS requires 216 lines of Java, not including whitespace or comments [25]. In Bloom ${ }^{L}$, vector clocks follow directly from the composition of the lmap and lmax lattices, and the entire KVS requires less than 100 lines of Ruby and Bloom ${ }^{L}$ code, including the client library. The ldom lattice requires an additional 50 lines of Ruby but is completely generic, and could be included as a built-in lattice.

\subsection{Quorum Reads and Writes}

To further demonstrate the flexibility of our implementation, we add an additional feature to our KVS: the ability to submit reads and writes to a configurable number of nodes. If a client reads from $R$ nodes and writes to $W$ nodes in a KVS with $N$ replicas, the user can set $R+W>N$ to achieve behavior equivalent to a quorum replication system [18], or use smaller values of $R$ and $W$ if eventual

\footnotetext{
${ }^{7}$ The observation that vector time has a lattice structure was made by Mattern [28].

${ }^{8}$ If the user stores a value that does not have a natural merge function, similar systems typically provide a default merge function that collects conflicting updates into a set for eventual manual resolution by the user. Such a strategy corresponds to using lset as the value lattice in Bloom ${ }^{L}$.
} 


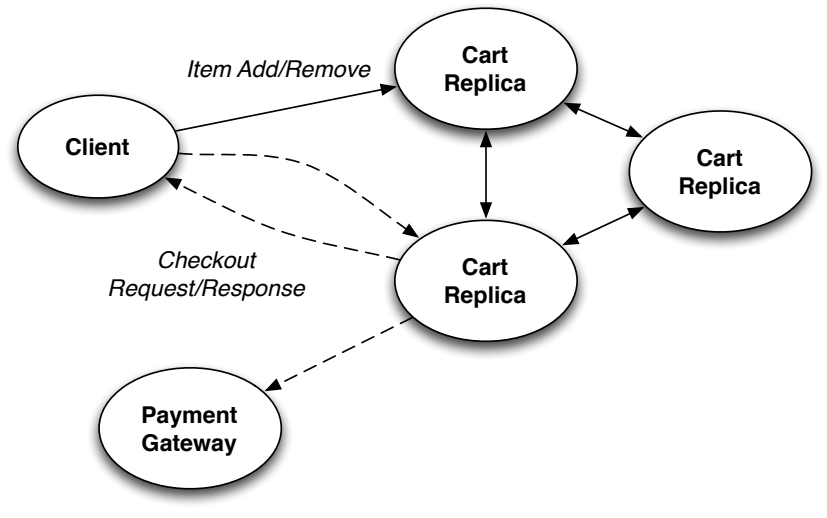

Figure 9: Shopping cart system architecture.

consistency is sufficient. This scheme allows users to vary $R$ and $W$ on a per-operation basis, depending on their consistency, durability, and latency requirements.

To support this feature, we can use the Bloom ${ }^{L}$ quorum voting pattern introduced in Figure 3. After sending a write to $W$ systems, the KVS client accumulates kvput_resp messages into an lset. Testing for quorum can be done in a monotonic fashion by mapping the lset to an lmax (using the size method) and then performing a threshold test using gt_eq on Imax. As expected, this is monotonic: once quorum has been reached, it will never be retracted.

Quorum reads work similarly except that the client must also merge together the $R$ versions of the record it receives. This follows naturally from the discussion in Section 5.2: the client simply takes the least upper bound of the values it receives, which produces the expected behavior. The client can optionally write the merged value back to the KVS (so-called "read repair" [12]); note that the ldom merge method also updates the record's vector clock appropriately.

\section{CASE STUDY: SHOPPING CARTS}

In the previous section, we showed how a complete, consistent distributed program can be composed via monotonic mappings between simple lattice types. In this section, we describe how Bloom ${ }^{L}$ overcomes the "type dilemma" of Bloom. In prior work, we introduced a case study in Bloom that seemed to require coordination because of the use of distributed aggregation [2]. By using custom lattice types, the Bloom ${ }^{L}$ CALM analysis can verify that our revised design is eventually consistent without need for coordination.

Figure 9 depicts a simple e-commerce system in which clients interact with a shopping cart service by adding and removing items over the course of a shopping session. The cart service is replicated to improve fault tolerance; client requests can be routed to any server replica. Eventually, a client submits a "checkout" operation, at which point the cumulative effect of their shopping session should be summarized and returned to the client. In a practical system, the result of the checkout operation might be presented to the client for confirmation or submitted to a payment processor to complete the e-commerce transaction. This case study is based on the cart system from Alvaro et al. [2], which was in turn inspired by the discussion of replicated shopping carts in the Dynamo paper [12].

\subsection{Monotonic Checkout}

Alvaro et al. argue that processing a checkout request is nonmonotonic because it requires aggregation over an asynchronously computed data set-in general, coordination might be required to ensure that all inputs have been received before the checkout re-

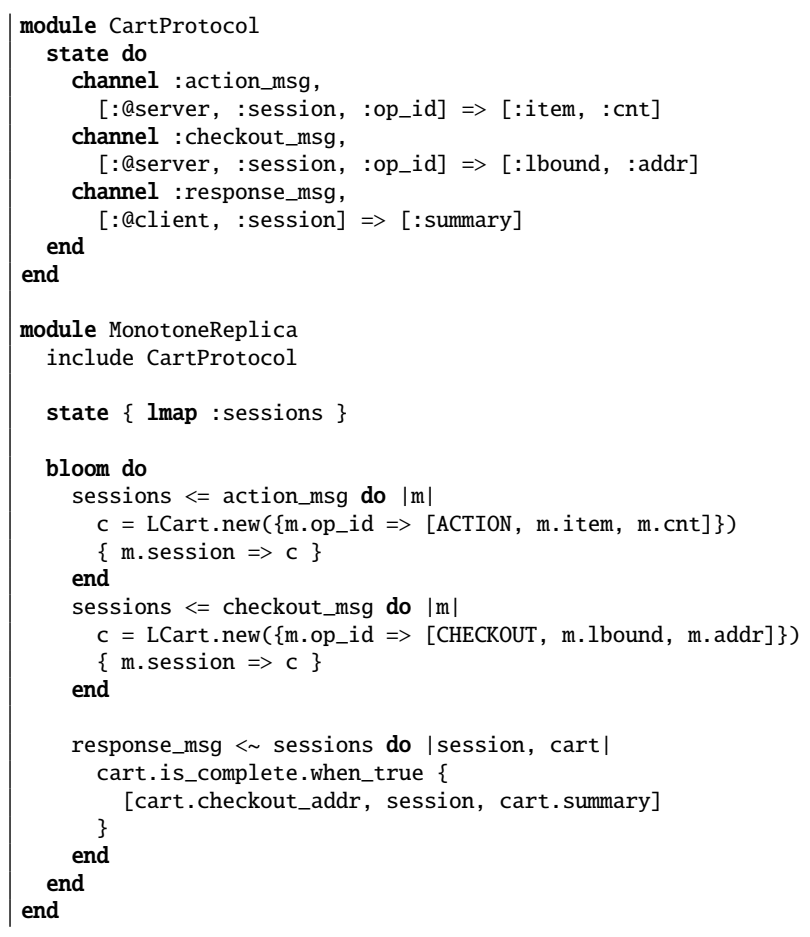

Figure 10: Cart server replica in Bloom $^{L}$ that supports a monotonic (coordination-free) checkout operation.

sponse can be returned to the client. However, observe that the client knows exactly which add and remove operations should be reflected in the result of the checkout. If that information can be propagated to the cart service, any server replica can decide if it has enough information to safely process the checkout operation without needing additional coordination. This design is monotonic: once a checkout response is produced, it will never change or be retracted. Our goal is to translate this design into a monotonic Bloom ${ }^{L}$ program.

Figure 10 contains the server code for this design (we omit the client code for the sake of brevity). Communication with the client occurs via the channels declared in the CartProtocol module. Each server replica stores an lmap lattice that associates session IDs with lcart lattice elements. An lcart is a custom lattice that represents the state of a single shopping cart. An lcart contains a set of client operations. Each operation has a unique ID; operation IDs are assigned by the client in increasing numeric order without gaps. An lcart contains two kinds of operations, actions and checkouts. An action describes the addition or removal of $k$ copies of an item from the cart. An lcart contains at most one checkout operation-the checkout specifies the smallest operation ID that must be reflected in the result of the checkout, along with the address where the checkout response should be sent. The lcart merge function takes the union of the operations in both input carts (operation IDs ensure idempotence). In Figure 10, lines 19 and 23 construct lcart elements that contain a single action or checkout operation, respectively. These singleton carts are then merged with the previous lcart associated with the client's session, if any.

An lcart is complete if it contains a checkout operation as well as all the actions in the ID range identified by the checkout. Hence, testing whether an lcart is complete is a monotone function: it is similar to testing whether an accumulating set has crossed a threshold. Hence, if any server replica determines that it has a complete 
cart, it can send a response to the client without risking inconsistency. ${ }^{9}$ Because this program contains only monotonic operations, CALM analysis can verify that this design is consistent without requiring additional coordination.

Note that the statement that produces a response to the client (lines 27-31) is contingent on having a complete cart. The monotone summary method returns a summary of the actions in the cartan exception is raised if summary is called on an incomplete cart. Similarly, attempting to construct an "illegal" lcart instance (e.g., an lcart that contains multiple checkout operations or actions that are outside the ID range specified by the checkout) also produces an exception, since this likely indicates a logic error in the program.

\subsection{Discussion}

This design is possible because a single client has complete knowledge of the shopping actions in its associated session. Hence, there is no need for additional distributed coordination - the server replicas accumulate knowledge but do not contribute new information themselves. If multiple clients could operate on a single shopping cart, some form of coordination between clients would be needed to ensure a consistent checkout result.

Note that the threshold test for completeness is a crucial part of this design. Until a cart is complete, its content changes in a "non-monotonic" fashion as items are added and removed. However, these non-monotonic changes are hidden inside the lcart type and are not directly visible to clients. Clients can only observe the cart's state once the cart is complete; at that point, the cart state is immutable and hence will not change in a non-monotonic fashion. Bloom $^{L}$ enables lcart to expose a limited "safe" interface and to hide transient non-monotonic changes from direct visibility.

\section{RELATED WORK}

This paper relates to work on concurrency control, distributed storage, parallel programming, and non-monotonic logic.

Semantics-based concurrency control: The traditional correctness criteria for concurrency control schemes is serializability [31]. However, ensuring serializability can be prohibitively expensive, for instance when transactions are long-running or the nodes of a distributed database are connected via an unreliable network. Thus, many methods have been proposed to allow non-serializable schedules that preserve some semantic notion of correctness. In particular, several schemes allow users to specify that certain operations can be commuted with other operations; this enlarges the space of legal schedules, increasing the potential for concurrency [14, 16, 44].

O'Neil describes a method for supporting "escrow" transactions, which allow operations that are only commutative when a certain limited resource is available [29]. For example, credits and debits to a bank account might only commute if the bank account balance can be guaranteed to be non-negative. We are currently exploring how to add support for escrow operations to Bloom ${ }^{L}$.

To support concurrent editing of shared documents, the groupware community has studied a family of algorithms known as Operational Transformations (OT) [13, 39]. Many OT algorithms have been proposed but the correctness criteria is typically familiar: after quiescence, all replicas of the document should converge to the same final state, the causal relationship between operations should be preserved, and the final state of the document should reflect the semantic intent of each user's editing operations [40].

\footnotetext{
${ }^{9}$ Without coordination, the client might receive multiple responses but they will all reflect the same cart contents.
}

Commutativity in distributed systems: Many distributed systems allow users to specify that certain operations are commutative, associative, or idempotent. Helland and Campbell observe that using commutative, associative and idempotent operations is particularly valuable as systems scale and guaranteeing global serializability becomes burdensome [19]. Many distributed storage systems allow users to provide "merge functions" that are used to resolve writewrite conflicts between replicas, allowing the system to eventually reach a consistent state (e.g., [21, 26, 33, 41]).

Principled eventual consistency: Shapiro et al. recently proposed Conflict-free Replicated Data Types (CRDTs), a framework for designing eventually consistent data values $[36,37]$. There are two kinds of CRDTs: "operation-based" types (called CmRDTs) and "state-based" types (called $C v R D T s$ ). Bloom ${ }^{L}$ is more closely related to CvRDTs (a CvRDT must be a semilattice), although the two CRDT variants are equivalently expressive. CRDTs and Bloom ${ }^{L}$ lattice types often use similar design patterns to achieve coordinationfree convergence. Unlike Bloom ${ }^{L}$, the CRDT approach considers the correctness of replicated values in isolation. This allows CRDTs to be more easily adapted into standalone libraries (e.g., Statebox [21]). However, the narrow focus of CRDTs means that, even if a CRDT is correct, application state may remain inconsistent (Example 1).

Burckhardt et al. advocate using revision diagrams to simplify the design of eventually consistent distributed programs [10]. A revision represents a logical replica of system state; revisions can be forked and joined, which represents creating new (logical) replicas and merging replicas together, respectively. Revision $a$ can only be joined to revision $b$ if $b$ is a descendant of the revision that forked $a$; this constraint ensures that revision graphs are semilattices. Note that this use of lattices differs from Bloom ${ }^{L}$ : whereas we constrain how data values can change, Burckhardt et al. constrain how replicas can synchronize their states. Another difference is that Burckhardt et al. allow non-deterministic outcomes, whereas we focus on confluence. In subsequent work, Burckhardt et al. have also proposed using abstract data types to encapsulate distributed state [11], although it is unclear whether their language allows user-defined types.

Parallel programming: Lattices have also been applied to parallel programming languages. Kuper and Newton recently proposed $\lambda_{\mathrm{par}}$, a deterministic parallel variant of the untyped $\lambda$-calculus [23]. Their approach guarantees deterministic program outcomes in the face of concurrent reads and writes to shared state by restricting reads and allowing only monotonically increasing writes to shared variables. Restricted reads in $\lambda_{\text {par }}$ are expressed by supplying a query set of lattice elements; a read either blocks or returns a unique element from the query set that is smaller than or equal to the current value of the shared variable (according to the lattice's partial order). This approach to restricting reads is somewhat similar to the use of threshold tests in Bloom ${ }^{L}$.

Extending monotonicity in deductive databases: Adding nonmonotonic operators to Datalog increases the expressiveness of the language but introduces significant complexities: unrestricted use of non-monotonicity would allow programs that imply logical contradictions (e.g., " $p$ if $\neg p$ "). A simple solution is to disallow recursion through aggregation or negation, which admits only the class of "stratified programs" [6]. Many attempts have been made to assign a semantics to larger classes of programs (e.g., [17, 34, 42]).

In addition to assigning a semantics to programs containing nonmonotonic operators, there has also been research on expanding the class of operators considered to be monotone. The prior work most in the spirit of Bloom ${ }^{L}$ is by Ross and Sagiv on monotonic aggregation [35]. Their framework is broader than the term "aggregation" would suggest. They actually look generally at monotone functions 
between partially ordered domains, which conceptually includes our lattice least upper bounds, monotone functions and morphisms (though they do not make those distinctions). They extend the notion of monotone programs to include "growth" by substituting a larger element in an atom for a smaller one. As we do, they require the lattice-valued columns to be functionally dependent on the other attributes in a predicate. One of their main results is that if the target domain of a monotone function is a complete lattice, then a program that uses such a function has a unique least fixpoint. While Bloom ${ }^{L}$ has much in common with Ross and Sagiv, there are several differences. They want to expand the collection of monotone programs but do not consider confluent distributed programming. In particular, they do not exploit the idempotence of least upper bound (since their source domain need not be a lattice), which is important to us, since it gives confluence with "at-least-once" message delivery. Also, they do not single out monotone functions that are actually morphisms. Hence they do not realize the evaluation flexibility we get from such functions. Finally, they do not propose a practical programming language or a framework that allows user-defined lattices to be composed safely.

Köstler et al. investigate deduction in the presence of a partial ordering of ground atoms, as might be induced by a lattice ordering on predicate columns [22]. They focus on reduced interpretations and models, where any atom dominated by another atom is discarded (unless it belongs to an unbounded maximal chain). One difference between their approach and ours is that reduced models need not satisfy the key dependencies we employ, because a pair of atoms that agree on a key need not have a common least upper bound. Köstler et al. construct a complete lattice over equivalence classes of reduced interpretations. However, the least upper bound of a set of models in this lattice may be infinite, even if each of those models is finite. We suspect our approach and theirs may be isomorphic when the monotone ordering on atoms is induced by lattice-valued columns, where the lattices have no infinite chains. However, we have not rigorously established this relationship yet.

The approach of Zaniolo and Wang [45] to aggregates in $\mathcal{L D} \mathcal{L}++$ is quite distinct from that of Ross and Sagiv, and, hence, from ours. Zaniolo and Wang do not use a lattice as the target domain for an aggregate or other kind of merge function. Rather, they achieve a kind of monotonicity by having an aggregate compute a series of partial results, rather than a single final result. With their formulation, a program computing an average would be judged monotone, where it would not for us. There are several reasons their treatment is not suitable for our purposes. For one, they point out that their programs are not monotone with the inclusion of a predicate for a final result. They do show examples where the partials alone suffice, for example, where the result of an increasing aggregate, such as sum, is compared to an upper bound. However, we see no machinery that would automatically distinguish such a use from an unsafe one, say with average. Further, their use of the choice() construct to assign an order to a set so an aggregate function can iterate over it poses two problems for us. One is that they allow different orders to yield different aggregate results; this does not fit with our goal of confluence. More problematic is that in a distributed computation, choice() would require communication to obtain a consistent order at different nodes, which goes against our desire for different sites to arrive at the same answer without coordination.

\section{DISCUSSION AND FUTURE WORK}

A key aspect of Bloom ${ }^{L}$ is that it enables the composition of consistent components. Rather than reasoning about the consistency of an entire application, the programmer can instead ensure that individual lattice methods satisfy local correctness properties (e.g., commutativity, associativity, and idempotence). CALM analysis verifies that when these modules are composed to form an application, the complete program satisfies the desired consistency properties.

Nevertheless, designing a correct lattice can still be difficult. To address this, we plan to develop tools to give programmers more confidence in the correctness of lattice implementations. For example, we plan to build a test data generation framework that can efficiently cover the space of possible inputs to lattice merge functions, drawing upon recent work on test generation for Bloom [3]. We also plan to explore a restricted DSL for implementing lattices, which would make formal verification of correctness an easier task.

Eventually consistent designs often consume more resources over time, requiring periodic "garbage collection" to restore efficiency [36]. Such distributed garbage collection protocols are typically application-specific and can be tricky to implement correctly. We are investigating ways in which $B \operatorname{loom}^{L}$ could assist programmers in writing garbage collection schemes. For example, a compiler analysis could verify that the garbage collection protocol does not change observable program behavior. Garbage collection might also have a natural representation in lattice-theoretic terms. For example, a common property is that once an operation has been applied by all replicas, information required to commute that operation with concurrent operations can be discarded. In lattice-theoretic terms, this could be represented by observing that, for a fixed set of replicas, the greatest lower bound of a lattice among the replicas increases monotonically; lattice elements that fall below the common greatest lower bound could be represented more efficiently.

In this paper, we have focused on programming with monotonically increasing values. In fact, many distributed programs feature values that increase monotonically for a period but then become immutable. For example, the lcart lattice described in Section 6 accumulates updates but then eventually becomes "complete" and stops changing. Once a value is immutable, any function can safely be applied to it (whether monotone or not) without risking inconsistency. The lcart (and lbool) lattices demonstrate that Bloom ${ }^{L}$ can represent such "monotonic-then-immutable" values, but we suspect that supporting immutability more directly might be useful. For example, a compiler analysis proving that a value is immutable in a certain situation would allow non-monotonic functions to safely be applied to it. Some immutable values can also be represented more efficiently: for example, a complete lcart need only store the "summarized" cart state, not the log of client operations.

\section{CONCLUSION}

In this paper, we proposed Bloom ${ }^{L}$, a distributed variant of Data$\log$ that extends logic programming with join semilattices. Bloom ${ }^{L}$ is particularly valuable for enabling coordination-free, consistent distributed programming, overcoming key hurdles in prior work. Like CvRDTs, Bloom $^{L}$ allows application-specific notions of "progress" to be represented as lattices and goes further by enabling safe mappings between lattices. Bloom ${ }^{L}$ improves upon our own earlier work by expanding the space of recognizably monotonic programs, allowing more programs to be verified as eventually consistent via CALM analysis. In addition to providing richer semantic guarantees than previous approaches, in our experience $B \mathrm{Bloom}^{L}$ provides a natural and straightforward language for building distributed systems.

\section{Acknowledgments}

We would like to thank Martin Abadi, Emily Andrews, Peter Bailis, Daniel Bruckner, Mosharaf Chowdhury, Tyson Condie, Sean Cribbs, Alan Fekete, Ali Ghodsi, Jonathan Harper, Leo Meyerovich, Josh Rosen, Evan R. Sparks, Matei Zaharia, and the anonymous re- 
viewers for their helpful feedback on this paper. This work was supported by the Air Force Office of Scientific Research (grant FA95500810352), the Natural Sciences and Engineering Research Council of Canada, the National Science Foundation (grants CNS0722077, IIS-0713661, IIS-0803690, and IIS-0917349), and gifts from EMC, Microsoft Research, and NTT Multimedia Communications Laboratories.

\section{REFERENCES}

[1] P. Alvaro, T. J. Ameloot, J. M. Hellerstein, W. R. Marczak, and J. den Bussche. A declarative semantics for Dedalus. Technical Report UCB/EECS-2011-120, EECS Department, UC Berkeley, Nov. 2011.

[2] P. Alvaro, N. Conway, J. M. Hellerstein, and W. R. Marczak. Consistency analysis in Bloom: a CALM and collected approach. In CIDR, 2011.

[3] P. Alvaro, A. Hutchinson, N. Conway, W. R. Marczak, and J. M. Hellerstein. BloomUnit: Declarative testing for distributed programs. In DBTest, 2012.

[4] P. Alvaro, W. R. Marczak, N. Conway, J. M. Hellerstein, D. Maier, and R. Sears. Dedalus: Datalog in time and space. In Datalog Reloaded. Springer Berlin / Heidelberg, 2011.

[5] T. J. Ameloot, F. Neven, and J. Van den Bussche. Relational transducers for declarative networking. In PODS, 2011.

[6] K. R. Apt, H. A. Blair, and A. Walker. Towards a theory of declarative knowledge. In J. Minker, editor, Foundations of Deductive Databases and Logic Programming, pages 89-148. Morgan Kaufmann, 1988.

[7] I. Balbin and K. Ramamohanarao. A generalization of the differential approach to recursive query evaluation. Journal of Logic Programming, 4(3):259-262, 1987.

[8] K. Birman, G. Chockler, and R. van Renesse. Toward a cloud computing research agenda. SIGACT News, 40(2):68-80, 2009.

[9] Bloom programming language. http: //www. bloom-lang.org.

[10] S. Burckhardt, M. Fähndrich, D. Leijen, and M. Sagiv. Eventually consistent transactions. In ESOP, 2012.

[11] S. Burckhardt, M. Fähndrich, D. Leijen, and B. P. Wood. Cloud types for eventual consistency. In ECOOP, 2012.

[12] G. DeCandia, D. Hastorun, M. Jampani, G. Kakulapati, A. Lakshman, A. Pilchin, S. Sivasubramanian, P. Vosshall, and W. Vogels. Dynamo: Amazon's highly available key-value store. In SOSP, 2007.

[13] C. A. Ellis and S. J. Gibbs. Concurrency control in groupware systems. In SIGMOD, 1989.

[14] A. A. Farrag and M. T. Özsu. Using semantic knowledge of transactions to increase concurrency. ACM TODS, 14(4):503-525, Dec. 1989.

[15] C. J. Fidge. Timestamps in message passing systems that preserve the partial ordering. In Australian Computer Science Conference, 1988.

[16] H. Garcia-Molina. Using semantic knowledge for transaction processing in a distributed database. ACM TODS, 8(2):186-213, June 1983.

[17] M. Gelfond and V. Lifschitz. The stable model semantics for logic programming. In ICLP, 1988.

[18] D. K. Gifford. Weighted voting for replicated data. In SOSP, 1979.

[19] P. Helland and D. Campbell. Building on quicksand. In CIDR, 2009.
[20] J. M. Hellerstein. The Declarative Imperative: Experiences and conjectures in distributed logic. SIGMOD Record, 39(1):5-19, 2010.

[21] B. Ippolito. statebox, an eventually consistent data model for Erlang (and Riak). http://labs . mochimedia.com/ archive/2011/05/08/statebox/, 2011.

[22] G. Köstler, W. Kießling, H. Thöne, and U. Güntzer. Fixpoint iteration with subsumption in deductive databases. Journal of Intelligent Information Systems, 4(2):123-148, 1995.

[23] L. Kuper and R. R. Newton. A lattice-theoretical approach to deterministic parallelism with shared state. Technical Report TR702, Indiana University, July 2012.

[24] L. Lamport. The part-time parliament. ACM TOCS, 16(2):133-169, May 1998.

[25] LinkedIn, Inc. Voldemort vector clock class. https: //raw.github.com/voldemort/voldemort/master/ src/java/voldemort/versioning/VectorClock . java. Accessed February 20, 2012.

[26] W. Lloyd, M. J. Freedman, M. Kaminsky, and D. G. Andersen. Don't settle for eventual: Scalable causal consistency for wide-area storage with COPS. In SOSP, 2011.

[27] W. R. Marczak, P. Alvaro, N. Conway, J. M. Hellerstein, and D. Maier. Confluence analysis for distributed programs: A model-theoretic approach. In Datalog 2.0, 2012.

[28] F. Mattern. Virtual time and global states of distributed systems. In Workshop on Parallel and Distributed Algorithms, 1989.

[29] P. E. O'Neil. The Escrow transactional method. ACM TODS, 11(4):405-430, Dec. 1986.

[30] Oracle Corporation. Streams Conflict Resolution. http://docs.oracle.com/cd/B10501_01/server. 920/a96571/conflict.htm.

[31] C. H. Papadimitriou. The serializability of concurrent database updates. Journal of the ACM, 26(4):631-653, Oct. 1979.

[32] D. S. Parker, G. J. Popek, G. Rudisin, A. Stoughton, B. J. Walker, E. Walton, J. M. Chow, D. Edwards, S. Kiser, and C. Kline. Detection of mutual inconsistency in distributed systems. IEEE Transactions on Software Engineering, SE-9(3):240-247, 1983.

[33] P. Reiher, J. Heidemann, D. Ratner, G. Skinner, and G. Popek. Resolving file conflicts in the Ficus file system. In USENIX Summer Conference, 1994.

[34] K. A. Ross. Modular stratification and magic sets for DATALOG programs with negation. In PODS, 1990.

[35] K. A. Ross and Y. Sagiv. Monotonic aggregation in deductive databases. In PODS, 1992.

[36] M. Shapiro, N. Preguiça, C. Baquero, and M. Zawirski. A comprehensive study of convergent and commutative replicated data types. Technical Report RR-7506, INRIA, 2011.

[37] M. Shapiro, N. Preguiça, C. Baquero, and M. Zawirski. Conflict-free replicated data types. In 13th International Symposium on Stabilization, Safety, and Security of Distributed Systems, 2011.

[38] SQLite Query Language: ON CONFLICT clause. http://sqlite.org/lang_conflict.html.

[39] C. Sun and C. Ellis. Operational transformation in real-time group editors: issues, algorithms, and achievements. In $C S C W$, 1998.

[40] C. Sun, X. Jia, Y. Zhang, Y. Yang, and D. Chen. Achieving convergence, causality preservation, and intention 
preservation in real-time cooperative editing systems. $A C M$ TOCHI, 5(1):63-108, Mar. 1998.

[41] D. B. Terry, M. M. Theimer, K. Petersen, A. J. Demers, M. J. Spreitzer, and C. H. Hauser. Managing update conflicts in Bayou, a weakly connected replicated storage system. In SOSP, 1995.

[42] A. Van Gelder, K. A. Ross, and J. S. Schlipf. The well-founded semantics for general logic programs. JACM, 38(3):619-649, 1991.
[43] W. Vogels. Eventually consistent. CACM, 52(1):40-44, 2009.

[44] W. E. Weihl. Commutativity-based concurrency control for abstract data types. IEEE Transactions on Computers, 37(12):1488-1505, 1988.

[45] C. Zaniolo and H. Wang. Logic-based user-defined aggregates for the next generation of database systems. In The Logic Programming Paradigm: Current Trends and Future Directions, pages 401-424. Springer Verlag, 1999. 